\title{
WiP Abstract: Diagnostics and Prognostics using Temporal Causal Models for Cyber Physical Energy Systems
}

\author{
Ajay Chhokra \\ Vanderbilt University \\ chhokraad@isis.vanderbilt.edu
}

\author{
Saqib Hasan \\ Vanderbilt University \\ saqibhasan@isis.vanderbilt.edu
}

\author{
Abhishek Dubey \\ Vanderbilt University \\ dabhishe@isis.vanderbilt.edu
}

\author{
Nagabhushan Mahadevan \\ Vanderbilt University \\ nag@isis.vanderbilt.edu
}

\author{
Gabor Karsai \\ Vanderbilt University \\ gabor@isis.vanderbilt.edu
}

\begin{abstract}
Reliable operation of cyber-physical systems such as Power Transmission and Distribution Systems is critical for the seamless functioning of a vibrant economy. These systems consist of tightly coupled physical (energy sources, transmission and distribution lines, and loads) and computational components (protection devices, energy management systems, etc). The protection devices such as distance relays help in preventing failure propagation by isolating faulty physical components. However, these devices rely on hard thresholds and local information, often ignoring system-level effects introduced by the distributed control algorithms. This leads to scenarios wherein a local mitigation in a subsystem could trigger a larger fault cascade, possibly resulting in a blackout. Efficient models and tool that curtail such systematic failures by performing fault diagnosis and prognosis are therefore necessary.
\end{abstract}

\section{CCS CONCEPTS}

-Hardware $\rightarrow$ Energy distribution; Power networks; Smart grid; •Computer systems organization $\rightarrow$ Reliability;

\section{KEYWORDS}

Diagnosis, Prognosis, Temporal Causal Diagram, Cyber Physical Energy Systems, Symbolic Mdoel Checking

\section{ACM Reference format:}

Ajay Chhokra, Saqib Hasan, Abhishek Dubey, Nagabhushan Mahadevan, and Gabor Karsai. 2017. WiP Abstract: Diagnostics and Prognostics using Temporal Causal Models for Cyber Physical Energy Systems. In Proceedings of The 8th ACM/IEEE International Conference on Cyber-Physical Systems, Pittsburgh, PA USA, April 2017 (ICCPS), 1 pages.

DOI: http://dx.doi.org/10.1145/3055004.3064843

Our approach uses Temporal Causal Diagrams (TCDs) to diagnose faults in cyber-physical energy systems. A Temporal Causal Diagram is a behavior augmented temporal failure propagation graph model. It is an amalgamation of Temporal Failure Propagation Graph (TFPG), that captures the propagation of failure in physical components and Timed Triggered Automaton (TTA) that

Permission to make digital or hard copies of part or all of this work for personal or classroom use is granted without fee provided that copies are not made or distributed for profit or commercial advantage and that copies bear this notice and the full citation on the first page. Copyrights for third-party components of this work must be honored. For all other uses, contact the owner/author(s).

ICCPS, Pittsburgh, PA USA

(C) 2017 Copyright held by the owner/author(s). 978-1-4503-4965-9/17/04.. $\$ 15.00$ DOI: http://dx.doi.org/10.1145/3055004.3064843 describes the behavior of discrete devices in nominal and faulty conditions. A TCD model relates the failure identification and propagation to different states of protection devices while accounting for constraints imposed by communication delays and operating conditions. The TCD model for a substation can be generated from the system topology involving transmission lines and protection devices. System TCD model is formed by connecting substation TCD models. The TCD based diagnosis system is hierarchal. The lower level uses local discrete event diagnosers, Observers, to hypothesize about the states and faults in physical and cyber components. A higher level reasoner produces system level hypothesis based upon the output of local observers. The approach does not involve complex real-time computations with high fidelity models, but reasons using efficient graph algorithms to explain the observed anomalies.

TCD prognostics uses two methodologies to predict fault progression. One method uses a two-step process wherein the first step involves traversing the failure graph from the fault to list secondary effects. The second step uses tools such as power flow solvers or demand response predictors to incorporate the changes resulting from the secondary effects into the TCD model. These steps are repeated until no secondary effects are seen or a blackout criterion is met. The convergence speed of this method depends upon the size of the graph and time taken by tools to complete their analysis at every step. The second method enables fast lookup of fault effect propagation by compiling the simulation results of a number of prescreened critical blackout causing component outages into compact data structures like Binary Decision Diagrams. TCD prognostics engine also suggests optimal system reconfiguration actions for arresting critical cascade causing component outages. The TCD diagnostics and prognostics approach is applicable to any resilient CPS that includes supervisory controllers that arrest propagation based upon local information without considering system-wide effects. The main contribution of our work lies in describing the TCD formalism, generation of TCD models from system specifications and presenting a robust diagnosis as well as prognosis engines. In order to show the validity of our approach, standard IEEE 14 bus system is used.

\section{ACKNOWLEDGMENTS}

This work is funded in part by the National Science Foundation under the award number CNS-1329803. The authors would like to thank Rishabh Jain, Dr.Srdjn Lukic (North Carolina State University) and Amogh Kulkarni (Vanderbilt University) for their help and discussions related to the work presented here. 